\title{
Superconducting RF Accelerating Cavity Developments
}

\author{
Evgeny Zaplatin \\ IKP, Forschungszentrum Juelich, D-52425 Juelich, Germany \\ e.zaplatine@fz-juelich.de
}

\begin{abstract}
For most practical accelerating rf cavities analytical solutions for the field distributions do not exist. Approximation by numerical methods provides a solution to the problem, reducing the time and expense of making and measuring prototype structures, and allowing rapid study and optimisation in the design stage. Many cavity design codes are available for sruding field distributions, interaction with charged particle beams, multipactor effects, stress analysis, etc. The types of calculations for different superconducting cavity design are discussed and illustrated by typical examples. The comparison of numerical simulations with some experimental results are shown.
\end{abstract}

\section{Introduction}

At present, many accelerators favour the use of superconducting (SC) cavities as accelerating RF structures[1]-[3]. For some of them, like long pulse Spallation Source or Transmutation Facility SC structures might be the only option. For the high energy parts of such accelerators the well-developed multi-cell elliptic cavities are the most optimal. For the low energy part the elliptic structures cannot be used because of their mechanic characteristics. The main working conditions of the SC cavities are as follow:

- Very high electromagnetic fields - maximum magnetic field on the inner cavity surface up to $B_{p k}=100 \mathrm{mT}$, maximum electric field on the inner cavity surface up to $E_{p k}=50 \mathrm{MV} / \mathrm{m}$. These high fields together with small cavity wall thickness $(2-4 \mathrm{~mm})$ result in the strong Lorenz forces which cause the wall deformations;

- Low temperature down to $2 \mathrm{~K}$, that again causes wall displacements after cool down;

- The pulse regime of operation that results in the addition requirements on cavity rigidity;

- High vacuum conditions $\left(10^{9}-10^{10}\right)$ and extra pressure on cavity walls from the helium tank also deform the cavity shape;

- High tolerances and quality surface requirements.

All deformations caused by these above mentioned reasons result in the working RF frequency shift in the range of hundreds Hz. Taking into account high 
Q-factor of SC cavities (the resonance bandwidth in the range of $\mathrm{Hz}$ ) such big frequency shift brings cavity out of operation. From the other hand, the use of any external tuning elements like plungers or trimmers are problematic as it results in the low down cavity acceleration efficiency. It means all these factors should be taken into account and complex electromagnetic simulations together with structural analysis should be provided during any real cavity design.

Here we present the scope of the possible RF accelerating structures, which can be used for different particle velocity $\beta=\mathrm{v} / \mathrm{c}$ starting from $\beta=0.09$ and ending with $\beta=1.0$. The considered structures are quarter-wave and half-wave coaxial cavities, spoke cavity and based on spoke cavity geometry multi-cell H-cavities and 5-cell elliptic cavities, which have been developed for various projects.

Because of their low power dissipation, SC structures do not need to be designed to maximaze the shunt impedance, and new designs, which would be inefficient for a normal conducting cavity can be explored. For example, SC resonators can be designed with much larger apertures that wouldn't be practical for normal conducting resonators. SC accelerators can also be built from very short structures, resulting in a increase in flexibility and reliability. Finally, SC resonators have the demonstrated capability of operating continuously at high accelerating fields. That's why the criterium of cavity geometry optimisation is to reach the maximal accelerating electric field.

\section{Elliptical Cavities}

Elliptical cavities are the most simple shape SC resonators (Fig. 1). They can be in a single or multi-cell configuration. The length of the cell is defined by the particle speed $\beta$ and cavity frequency $f-$ celllength $=\beta c / 2 f$. This defines the limitation on this type cavity use for low energy (low $\beta$ 's) particles - at and below $\beta=0.4-0.5$ the cell geometry becomes narrow which doesn't fulfill the mechanic requirements. Another limitation comes from the frequency side lower frequency bigger cavity. As the cavity is made out of rather expensive pure niobium, the lowest frequency which can be considered is about $350 \mathrm{MHz}$.

\subsection{Middle Cell Geometry Optimisation}

Usually, an elliptical cavity design is a compromise between various geometric parameters which should define a most optimal cavity shape in terms of an accelerator purpose (Fig. 1). Within a SC proton linac design there is a need of grouping of cavities with different $\beta=v / c$ values. It means that there should be several different cavities and the process of the accelerating structure design for SC linac becomes time consuming. The suggested in [4] procedure allows to get an optimal geometry optimisation leaving a freedom for the cavity parameters choice. As the cavity is strictly azimuthal symmetric these calculations can been done with a help of 2D cavity simulation code like SUPERFISH[5].

The main advantage of any SC cavity is a possibility of the high accelerating electric field $\left(E_{a c c}\right)$ creation. There are two characteristics which limit in principle an achievable value of $E_{a c c}$. They are the peak surface electric field $\left(E_{p k}\right)$, 

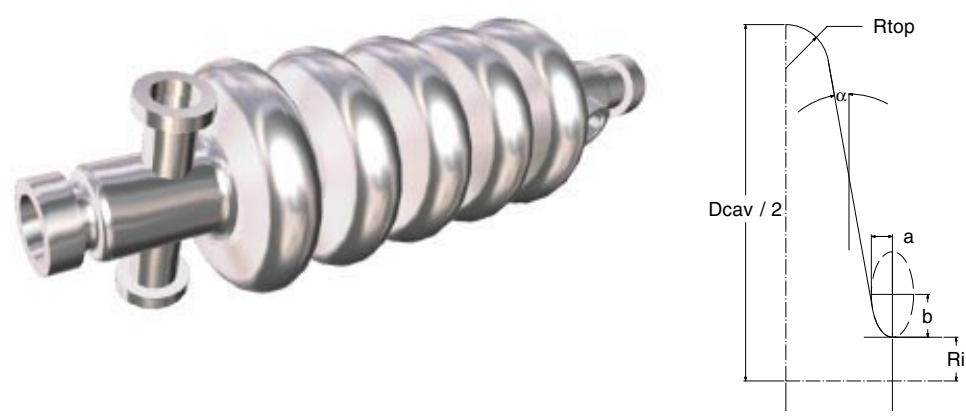

Fig. 1. Elliptical Cavity \& Cell Geometry (1/4 part is shown)

which is allocated around cavity iris and the peak surface magnetic field $\left(H_{p k}\right)$ in the cavity dome region. $H_{p k}$ is important because a superconductor will quench above the critical magnetic field. Thus $H_{p k}$ may not exceed this level. $E_{p k}$ is important because of the danger of field emission in high electric field regions. All these mean that to maximise the accelerating field first of all it is therefore important during a cavity design to minimise the ratios of peak fields to the accelerating field. There are some more figures of merit to compare different designs such as power dissipation $P_{c}$, a quality factor $\mathrm{Q}$ and shunt impedance $R_{s h}$. But these parameters are not so crucial to the cavity accelerating efficiency and may be varied in some limits without any sufficient harm for a system in whole.

One of the basic figure which will influence on the further cell geometry is a cell-to-cell coupling coefficient in multicell cavity. This parameter defines field flatness along the cavity. This characteristic is obtained in conjunction with beam dynamic calculations and more or less is defined as a first. In practices the usual value for the coupling is above $1 \%$.

Another cavity cell geometric limitation comes from the radius of the material curvature in the region of the cavity iris. The smallest radius estimated is to be 2-3 times bigger than a cavity wall thickness. An investigation of the plots presented on Figs. 2-3 with two limiting parameter characteristics crossing helps to make a proper choice of cell.

\subsection{Multipacting}

Making the cavity cell geometry optimisation one should take into account the possibility of a multipactor resonance discharge. At the moment several groups in the field had active programs or developments of programs that can be used to study potential multipacting effects in rf structures[6]-[8]. Three of the programs can be applied to $3 \mathrm{D}$ problems while the rest are applicable to $2 \mathrm{D}$ structures. Considering that the big fraction of SC cavities are rotationally symmetric, these $2 \mathrm{D}$ programs do still cover a wide range of interesting problems. Some of the groups are working on the extension of their codes to 3D capabilities. 

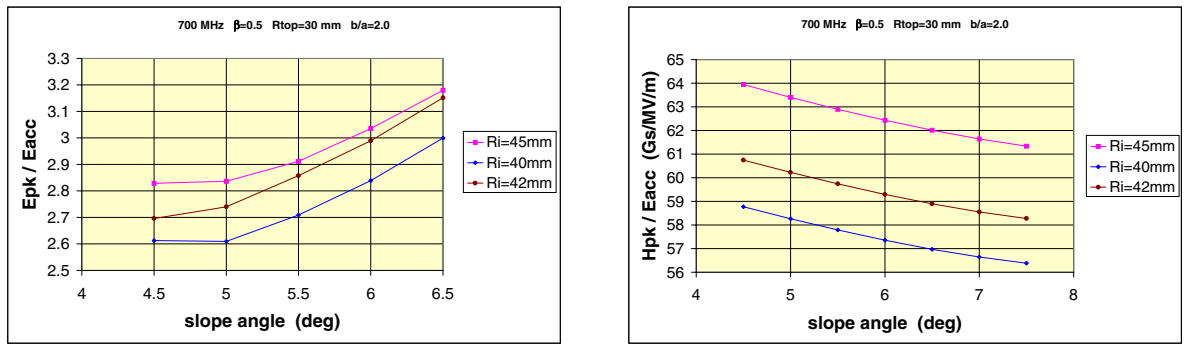

Fig. 2. $E_{p k} / E_{a c c} \& H_{p k} / E_{a c c}$ vs. Cavity Slope Angle $\alpha(\beta=0.5)$
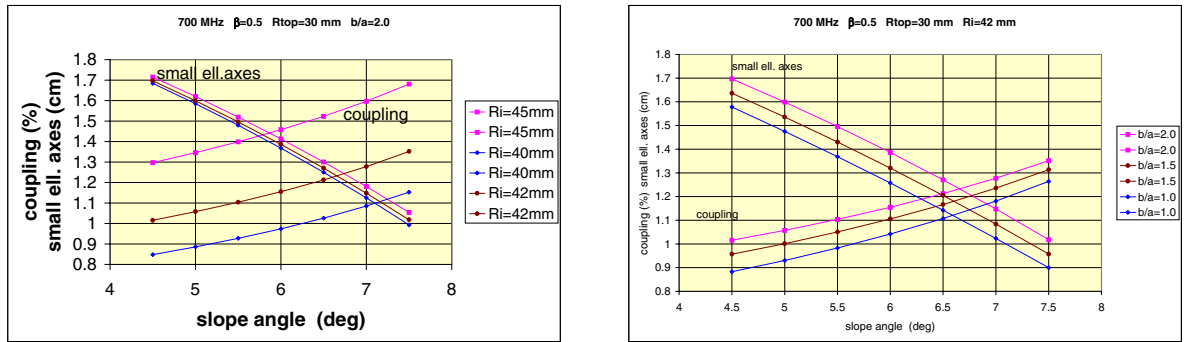

Fig. 3. Cell-to-Cell Coupling vs. Cavity Slope Angle $\alpha(\beta=0.5)$

Multipacting in rf structures is a resonant process in which a large number of electrons build up an multipacting, absorbing rf power so that it becomes impossible to increase the cavity fields by raising the incident power. The electrons collide with structure walls, leading to a large temperature rise and in the case of superconducting cavities to thermal breakdown. Basic tools of analysis of multipactor are counter functions. These functions are defined as follows. For a fixed field level a number of electrons are sent from different points on the cavity wall in different field phases and compute the electron trajectories. After an impact on the wall of cavity the trajectory calculation is continued if the phase of the field allows the secondary electrons to leave the wall. After a given number of impacts the number of free electrons (counter function) and total number of secondary electrons (enhanced counter function) are counted. Usually in elliptical cavity it happens around a dome equator region (Fig. 4). The proper cavity shape selection helps to avoid this resonance.

Here we present the results of such simulations[8] made by the Helsinki group for the same frequency $(700 \mathrm{MHz})$ and different $\beta=0.5-0.9$. On Fig. 4 the possible dangerous regions of $E_{a c c}$ are shown. The tendency is that the lower $\beta$ cavities because of their smaller dome radius are more affected by multipactor. 

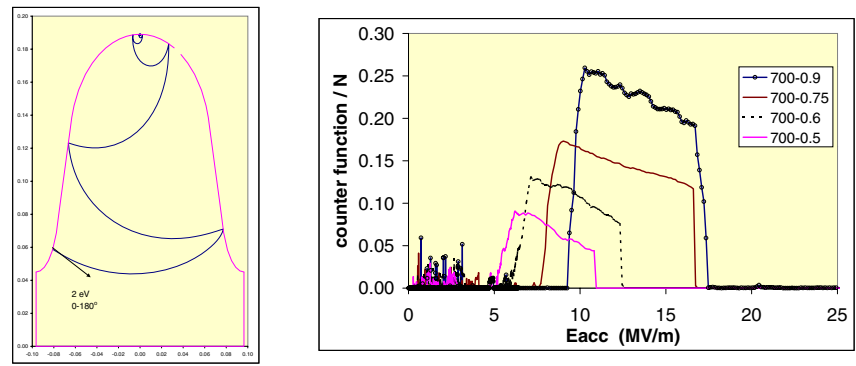

Fig. 4. Schematic drawing of multipactor resonance discharge \& multipactor dangerous regions for different elliptic cavities $(700 \mathrm{MHz}, \beta=0.5-0.9)$.

\subsection{Mechanics}

All superconducting rf resonators are niobium cavities that are enclosed within helium vessels. These vessels are filled with liquid helium that floods the cavities and maintain the $2 \mathrm{~K}$ operating temperature. Mechanical analysis consist of design calculations for all critical cavity assembly components, cavity tuning sensitivity analysis, active tuner and bench tuner load determination, cavity assembly cool-down analysis, natural frequency and random response analysis, inertia friction weld analysis and critical flaw size calculations.

As an example Fig. 5 presents results of cavity wall displacements caused by the extra pressure from the helium vessel. Similar deformations result from cooldown shape shrinking and the Lorenz forces originated by electromagnetic fields. To minimize the cavity deformation the stiffening rings are installed between cells still alowing enough cavity flexibility for structure tune.

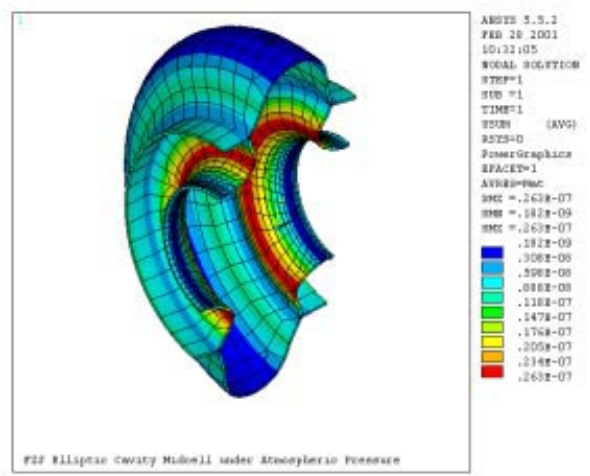

Fig. 5. Deformation of Elliptical Cavity Mid-Cell under Atmospheric Pressure 
High repetition rates like $50 \mathrm{~Hz}$ for ESS will require a close look to the mechanical resonances of the cavities. Mechanical resonances can influence the phase behaviour of the cavity during a pulse, which can hardly be compensated by a good control system, even if a lot of additional power is available. Additionally, cavity rf resonance is sensitive to vibrations of sub- $\mu \mathrm{m}$ amplitudes. These microphonic effects cause low frequency noice in the accelerating fields. Therefore, carefull mechanic eigen mode analysis of the cavity together with its enviroments should be conducted. Fig. 6 presents a technical drawing of 500 $\mathrm{MHz}, \beta=0.755$-cell elliptic cavity module of Forschungszentrum Juelich[3]. This facility is being used as an experimental installation for deep mechanic cavity property investigations. An experimental program comprises Lorenz force cavity detuning and its compensation and mechanical resonances and microphonic effect evaluations. On Fig. 6 a fast fourier transformation of the response to the time domain step function is shown. It reflects the first series of mechanical resonances of the structure. The dynamic analysis of the cavity with simplified cryoenviroments using ANSYS codes have been provided. The results are summarized in Table 1.
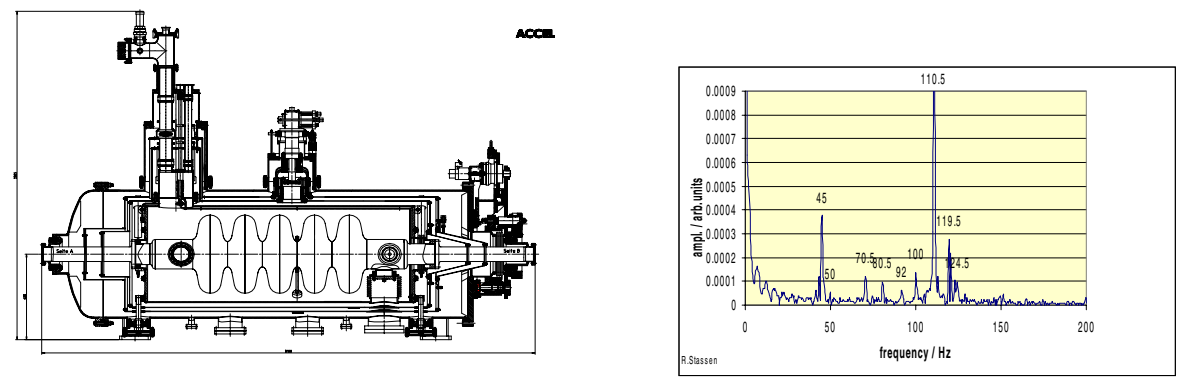

Fig. 6. FZJ Experimental Cryo Module with 5-cell Elliptic Cavity and Experimantal Results of Dynamic Modal Analysis

Table 1. FZJ Elliptic Cavity Dynamic Analysis with ANSYS

\begin{tabular}{|l|c|c|c|c|c|c|c|c|}
\hline mode & 1 & 2 & 3 & 4 & 5 & 6 & 7 & 8 \\
\hline frequency, $\mathrm{Hz}$ & 48.9 & 67.5 & 68.6 & 110.8 & 131.9 & 158.0 & 181.1 & 191.0 \\
\hline
\end{tabular}

\section{Low- $\beta$ Cavities}

This type of SC rf structures have been in use for nearly two decades in heavyion booster linacs. Because of the requirement of short accelerating structures, low operating frequencies are used to make the structures as long as possible, 
so that the accelerating voltage per structure is maximized. The difference in use is defined by the type of the particle which has to be accelerated. The very first restriction on the structure choice for this range of particle energies (up to $20 \mathrm{MeV}$ ) for light particle (protons, deuterons) acceleration comes from the beam dynamics. Because of the phase shift between RF field and particle owing to acceleration it is impossible to use the cavities with a number of gaps bigger than two. A single-gap, so-called reentrant cavity, or elliptic cavities are not the best choice for such low energy range and especially for pulse mode operation because of its bad mechanic characteristics. This defines the use of coaxial quarter(QWR) and half-wale length cavities for the $\beta$ up to 0.2 . The weak point of any QWR is its non-symmetry, which results in transversal components (especially magnetic) of rf field along the beam path, which is the serious problem for acceleration of light particles like protons. This can be eliminated by the half-wave cavity use. This type of structures comprises two well-known cavities, which are called coaxial half-wave resonator (HWR) and spoke cavity (Fig. 7). HWR is just a symmetric extension of quarter-wave cavity relative to the beam path. The simulation of this type of cavities require the use of real 3D codes, like for instance MAFIA[11].

\subsection{Half-Wave Length Cavities}

The range of half-wave structure application is for rather low $\beta \leq 0.2$ and fundamental frequency under $300 \mathrm{MHz}$. The resonant frequency is defined by the line length, inner-outer radius ratio and capacitance of an accelerating gaps. An accelerating field magnitude is limited by peak magnetic field that is defined mainly by the inner-outer electrode distance. This favours the use of the conical HWR (Fig. 7). The disadvantage of the cone cavity is its larger longitudinal extension. On the other hand this may be compensated by the cross-cavity installation.
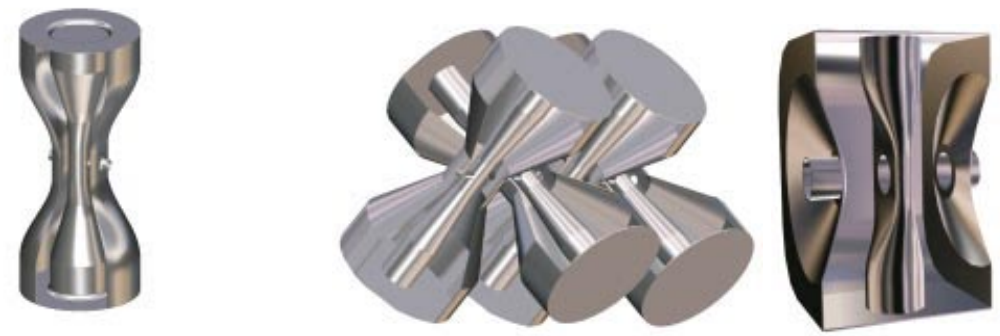

Fig. 7. Half-Wave Length Cavity, Cross-Cavity Installation \& Spoke Cavity.

Another accelerating structure for this range of particle energy is a spoke resonator (Fig. 7). The spoke cavity by definition is a coaxial half-wave length cavity with an outer conductor turned on ninety degrees so that its axe is directed along the beam path. An equivalent capacitance of such cavity is defined 
by the distance between conductors in the center of the cavity along this axe. A distribution of an electromagnetic field in such cavity is the same like in coaxial cavity. The range of application of this cavity is from 100 to $800 \mathrm{MHz}$ of fundamental frequency and $\beta=0.1-0.6$. The limitations of application are defined mainly by the resonance capacitance grow for low- $\beta$ values which in its turn reduces cavity diameter. The spoke cavity acceleration efficiency is defined by the magnetic field magnitude on the inner electrode surface like in the coaxial resonators. The comparison of cross-cavity half-wave conical resonator installation with the set of spoke cavities in terms of maximal reachable accelerating field favours the use of the first option (Fig. 8)[12].
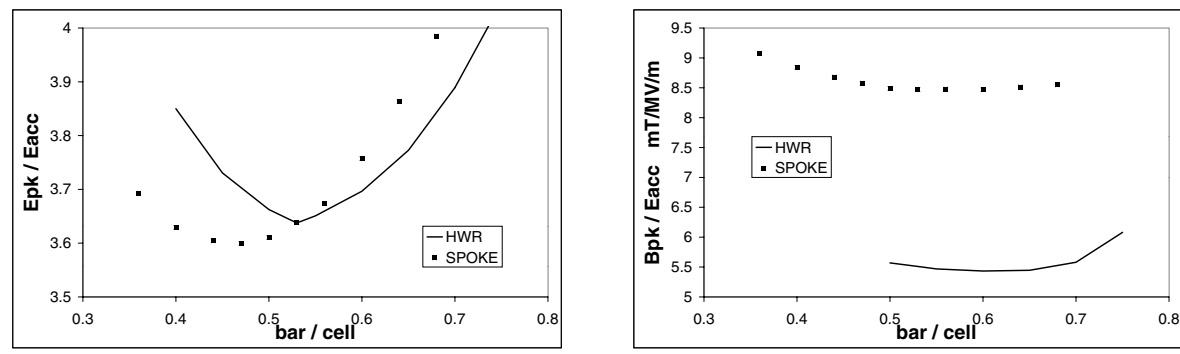

Fig. 8. Half-Wave Length Coaxial Cavity \& Spoke Cavity Comparison (MAFIA Simulations).

\subsection{Multi-Gap H-Cavity}

Starting with the value $\beta=0.2$ there is a possibility to use multi-gap (more than two) accelerating structures. Such structures could represent the same cylindrical or modified shape outer conductor loaded with several electrodes (Fig. 9). But as soon as one adds at least another spoke in such structure it turns from the coaxial spoke cavity into H-type cavity, what is defined by the electromagnetic field distribution. The detailed results of multy-gap H-cavity optimisation are published elsewhere[13]. The main design criterions are the same like for spoke cavity. For the cavity tune the deformation of the end plates is used, which equivalent to the last gap capacitance change. The results of the numerical simulations and model measurements for $700 \mathrm{MHz}, \beta=0.2$ 10-gap H-cavity are shown on Figs. 10-9. If to take into account that the whole cavity length is about $500 \mathrm{~mm}$ and end plate shift for tuning is within $\pm 1 \mathrm{~mm}$, the high precision of simulations can be achieved.

\subsection{Mechanics}

The same structural analysis of low- $\beta$ cavities has to be made to find the model predictions for peak stresses, deflections and flange reaction forces under vacuum 

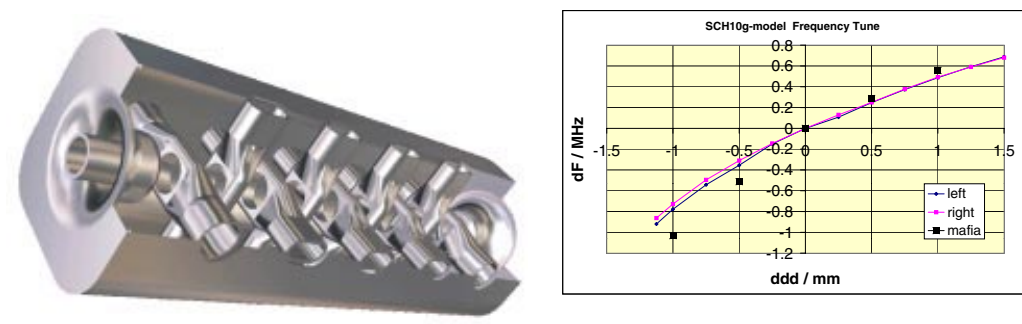

Fig. 9. 10-Gap H-Cavity and MAFIA Simulations \& Experimental Data of Structure Tune.
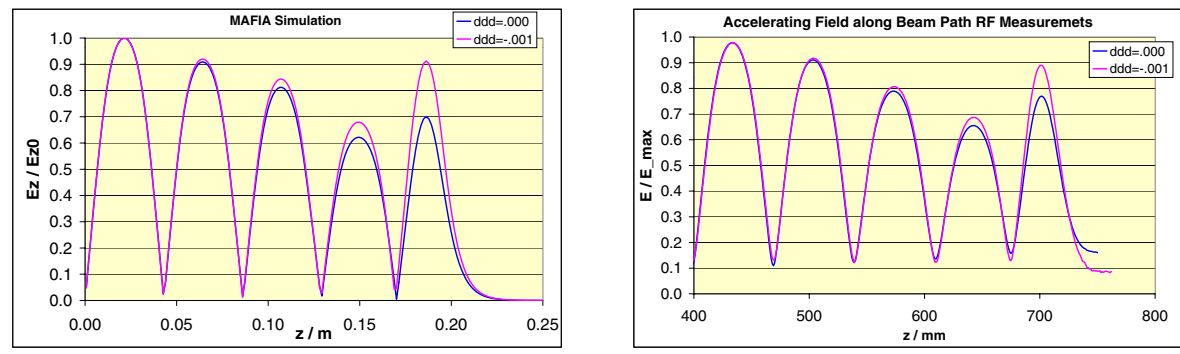

Fig. 10. MAFIA Simulation (left) and Experimental Results (right) of 10-Gap HCavity Electrical Field Distribution by Tuning

loads and room temperature, and also for forces required to produce a specify tuning deflection. The important part of simulations is devoted to the determination of resonant structural frequencies (Fig. 11). These structures differ from elliptic cavities by their more complicate $3 \mathrm{D}$ geometries that result in cavity higher rigidity and mechanical stability but complicate simulations. The main purpose of such calculations is as close as possible prediction of cavity frequency shift caused by structure deformations. This frequency shift should be later covered by cavity tuning range. The high structure frequency like $700 \mathrm{MHz}$ defines smaller cavity dimensions, which result in smaller deformations and bigger tuning range. For spoke and 10 -gap $\mathrm{H}$-cavities $(700 \mathrm{MHz}, \beta=0.2)$ the tuning range is within $1 \mathrm{MHz} / \mathrm{mm}$ which easily can cover the possible wall deformation effects. For the frequency $320 \mathrm{MHz}$ the tuning reduces down to $100-200 \mathrm{kHz} / \mathrm{mm}$. This numbers are usually used as the minimal tuning range that should be reached. The coaxial quarter- and half-wave cavities with frequency $160 \mathrm{MHz}$ can reach the same numbers. Making the proper structure design together with cryomodule the mechanical eigen resonances for all structures can be shifted well above $100 \mathrm{~Hz}$. 

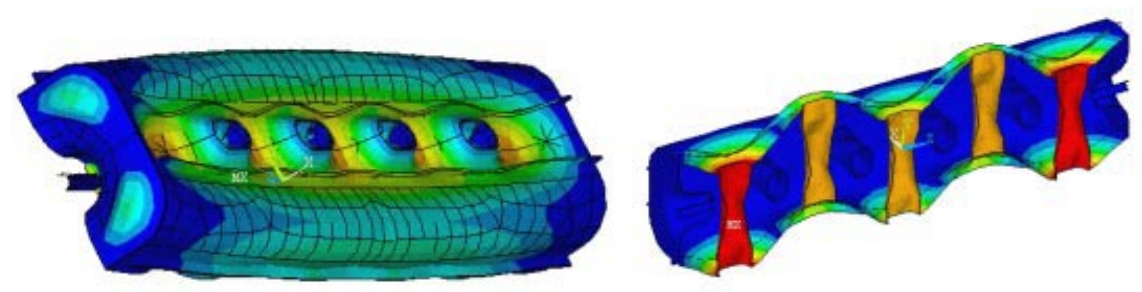

Fig. 11. 10-Gap H-Cavity under Extra Pressure and Modal Analysis.

\section{References}

1. F. L. Krawczyk et al.: Superconducting Cavities for the APT Accelerator. Proceedings of the 1997 Particle Acc. Conf., p. 2914.

2. D. Barni et al.: SC Beta Graded Cavity Design for a Proposed $350 \mathrm{MHz}$ LINAC for Waste Transmutation and Energy Production. Proceedings of the 1998 European Particle Acc. Conf., Stockholm, p.1870 (1998).

3. W. Bräutigam, et al.: Design considerations for the linac system of the ESS. NIM, B 161-163 (2000) 1148-1153.

4. E. Zaplatin: Design of Superconducting RF Accelerating Structures for High Power Proton Linac. ISSN 1433-559X, ESS 104-00-A, Juelich, July 2000.

5. J. H. Billen and L. M. Young: POISSON/SUPERFISH on PC Compatibles. Proceedings of the 1993 Particle Acc. Conf., Vol. 2, p. 790.

6. R. Parodi: Preliminary analysis of Multipacting Barriers in the $500 \mathrm{MHz}$ beta 0.75 ESS superconducting cavity. INFN, Genova, 2000.

7. S. Humphries Jr: Electron Multipactor Code for High-Power RF Window Development. Particle Accelerators, Vol. 62, pp. 139-163, 1999.

8. P. Ylä-Oijala: Multipacting Analysis for Five Elliptical Cavities. Rolf Nevanlinna Institute, University of Helsinki, Helsinki, September 22, 1999.

9. ANSYS is a trade mark of SAS IP, Inc., http://www.ansys.com

10. K. W. Shepard: Superconducting Heavy-Ion Accelerating Structures. Nuc. Instr. and Meth., A382 (1996) 125-131, North-Holland, Amsterdam.

11. M. Bartsch et al.: Solution of Maxwell's Equations. Computer Physics Comm., 72, 22-39 (1992).

12. E. Zaplatin et al.: Very Low- $\beta$ Superconducting RF Cavities for Proton Linac. ISSN 1433-559X, ESS 104-00-A, Juelich, July 2000.

13. E. Zaplatin: Low- $\beta$ SC RF Cavity Investigations. Workshop on RF Superconductivity SCRF2001, Tsukuba, Japan, 2001. 